\title{
An invariant subspace problem for multilinear operators on Banach spaces and algebras
}

John Emenyu*(0)

"Correspondence:
jemenyu@must.ac.ug
Department of Mathematics,
Faculty of Science, Mbarara
University of Science and
Technology, P.O. Box 1410, Mbarara,
Uganda

"Correspondence:

jemenyu@must.ac.ug

Department of Mathem Uganda

\begin{abstract}
This paper is concerned with the study of invariant subspace problems for nonlinear operators on Banach spaces/algebras. Our study reveals that one faces unprecedented challenges such as lack of vector space structure and unbounded spectral sets when tackling invariant subspace problems for nonlinear operators via spectral information. To bypass some of these challenges, we modified an eigenvalue problem for nonlinear operators to cater for the structural properties of nonlinear operators and then established that nonlinear operators of finite type on a complex Banach algebra have nontrivial invariant subspaces.
\end{abstract}

Keywords: Banach spaces; invariant subspaces; strongly invariant subspaces; multilinear and polynomial operators

\section{Introduction}

The aim of this paper is to study invariant subspace problems for polynomial and multilinear operators on infinite dimensional Banach spaces.

Throughout this paper, we denote Banach spaces by $E$ and $F$, and the dual space of $E$ by $E^{\prime}$. A map $T: E \times \cdots \times E \rightarrow F$ is $m$-linear if it is linear in each of the $m$-variables and for this map, a map $p: E \rightarrow F: p(x)=T(x, \ldots, x)$ for all $x \in E$ is called an $m$-homogeneous polynomial map. We denote the space of continuous multilinear and polynomial maps by $\mathcal{L}\left({ }^{m} E ; F\right)$ and $\mathcal{P}\left({ }^{m} E ; F\right)$, respectively, see $[1,2]$. The term operator will be restricted to the elements of $\mathcal{L}\left({ }^{m} E ; E\right)$; notice 1-linear operators are just elements of $\mathcal{L}(E)$.

Definition 1.1 Let $p \in \mathcal{P}\left({ }^{m} E ; E\right)$ be associated to $T \in \mathcal{L}\left({ }^{m} E ; E\right)$. A closed linear subspace $\mathcal{M}$ of $E$ is invariant for $p \in \mathcal{P}\left({ }^{m} E ; E\right)$ if $p(\mathcal{M}) \subseteq \mathcal{M}$; it is invariant for $T \in \mathcal{L}\left({ }^{m} E ; E\right)$ if $p(\mathcal{M}) \subseteq \mathcal{M}$; it is strongly invariant for $T \in \mathcal{L}\left({ }^{m} E ; E\right)$ if $T(\mathcal{M}, \ldots, \mathcal{M}) \subseteq \mathcal{M}$. The cases $\mathcal{M}=$ $\{0\}$ and $\mathcal{M}=E$ are called trivial subspaces.

Remark 1.2 Let $\mathcal{M}$ be a closed linear subspace of $E$. Let $p \in \mathcal{P}\left({ }^{m} E\right.$; $\left.E\right)$ be associated to $T \in \mathcal{L}\left({ }^{m} E ; E\right)$. The following are equivalent.

(i) $\mathcal{M}$ is invariant for $p \in \mathcal{P}\left({ }^{m} E ; E\right)$.

(ii) $\mathcal{M}$ is invariant for $T \in \mathcal{L}\left({ }^{m} E ; E\right)$.

Proof It is immediate from Definition 1.1.

(c) 2016 Emenyu. This article is distributed under the terms of the Creative Commons Attribution 4.0 International License (http://creativecommons.org/licenses/by/4.0/), which permits unrestricted use, distribution, and reproduction in any medium, provided you give appropriate credit to the original author(s) and the source, provide a link to the Creative Commons license, and indicate if changes were made. 
Problems 1.3 and 1.4 are referred to, in the sequel, as invariant subspace problem (ISP) and eigenvalue problem for multilinear operators, respectively.

Problem 1.3 Given a bounded operator $p \in \mathcal{P}\left({ }^{m} E ; E\right)$, does there exist a closed invariant subspace $\mathcal{M}$ of $E$ for $p \in \mathcal{P}\left({ }^{m} E ; E\right)$ besides $\mathcal{M}=\{0\}$ and $\mathcal{M}=E$ ?

Problem 1.4 Given a bounded operator $p \in \mathcal{P}\left({ }^{m} E ; E\right)$, does there exist a nonzero vector $v \in E$ such that $p(v)=\lambda v$ for some $\lambda \in \mathbb{k}$ ?

The notion of invariant subspaces introduced in [3] provided a basis for extending the underlying notion to nonlinear operators in a natural way; however, a natural entry point to delve into the study of such notion in infinite dimensions is first to deal with ideals of compact nonlinear maps generated by $\left\{T_{1} T_{2} \ldots T_{m}: T_{j} \in \mathcal{L}(E)\right\}$ where $m \in \mathbb{N}$ is fixed. These maps are known as maps of finite type and they inherit certain intrinsic properties of linear maps such as approximability by finite dimensional maps and bounded point spectra that are useful in the study of their associated ISPs. Notice $T \in \mathcal{L}\left({ }^{m} E ; F\right)$ is finite dimensional if its multirange $T(E, \ldots, E)$ is contained in a finite dimensional subspace of $F$; see Section 3 in [4]. In general, structural properties of $T_{j} \in \mathcal{L}(E)$ such as compactness were key in the study of their ISP; see [5, 6]. On the one hand, the work of Bényi and Torres [7] embodies different kinds of notions of compactness of a class of bilinear operators of finite type. Indeed, separate compactness of each section map of a bilinear map of finite type does not guarantee its compactness thus lending a study of Problem 1.3 a more general and broader consideration rather than restricting it to only compact nonlinear operators.

In Section 2, we will give a review of nonlinear spectral theory and Section 3 contains a review of techniques for tackling Problem 1.3, the modified form of Problem 1.4 and some tools such as Lemma 3.4 for establishing our results in Section 5. Section 4 stipulates problems associated with tacking Problem 1.3 whereas Section 5 comprises our major results.

\section{An overview of nonlinear spectral theory}

The study of Problem 1.3 via Problem 1.4 requires an in-depth knowledge of solvability properties of the equation $\lambda z-p(z)=0$, hence, the structural properties of $p \in \mathcal{P}\left({ }^{m} E ; E\right)$; namely, injectivity of $\lambda I-p$ and whether it maps some bounded neighborhood of 0 onto a neighborhood of 0 , continuity of $(\lambda I-p)^{-1}$, boundedness and nontriviality of the nullset $\mathcal{N}(\lambda I-p)$. These underlying structural properties yielded several variants of spectra of $p \in \mathcal{P}\left({ }^{m} E ; E\right)$, each having different notions of eigenvalues; see Chapters 6-9 in [8]. Among several others, the Furi-Martelli-Vignoli spectrum $\sigma_{\mathrm{FMV}}(p, I)$, see [9], 1978, and its modified form called Appell-Giorgieri-Väth spectrum $\sigma_{\mathrm{AGV}}(p, I)$, see [8], p.150, account for asymptotic properties of stably nonlinear solvable continuous operators; the Feng spectrum $\sigma_{F}(p, I)$ accounts for global properties of epi and $k$-epi operators, see [10], 1997; the small Väth spectrum $\phi(p, I)$ and large Väth spectrum $\Phi(p, I)$ account for local properties of strictly and properly epi operators, respectively, see [11], 2001. However, none of the known nonlinear spectra adheres to the minimal requirements; specifically most of their associated eigenvalue notions are incompatible with the general notion of the classical eigenvalues [12], Definitions 1 and 2. Further, these spectra may be disjoint from point spectra, see Example 6.6 in [8], [11], are not discrete and unbounded even if their under- 
lying operators are bounded and compact, see Example 3.5 in [12], Theorem 3.5 in [13]; [8] gives extensive and exhaustive literature on this topic.

\section{An overview of invariant subspace techniques for nonlinear maps}

An attempt of a de facto investigation of Problem 1.3 is by Donoghue, see Example 4 in [14] and [3], which comprise a more systematic study via Problem 1.4 or more generally via elementary invariant subspaces. The challenge to the underlying technique in [3] is the limitation in knowledge of topological degrees in infinite dimensions. Moreover, the nondiscreteness and disjointness of nonlinear spectra from point spectra hinder the adaptation of linear approaches to Problem 1.3. For instance, extending perturbation methods [15-17] modeled on the assumption $\sigma(T+K) \subset \sigma(T)$ is tantamount to the consideration of solvability properties of the eigenvalue equation $\lambda z-p(z)=0$ for some $\lambda \in \mathbb{k}$ or alternatively structural properties of $p \in \mathcal{P}\left({ }^{m} E ; E\right)$. This in turn is tantamount to the application of $\sigma_{\mathrm{FMV}}(p, I), \sigma_{\mathrm{AGV}}(p, I), \sigma_{F}(p, I)$ but due to the underlying problems they usually lead to either unsuccessful or partial solutions to the Problem 1.3; they are marginally successful in very limited circumstances such as when $p \in \mathcal{P}\left({ }^{m} E ; E\right)$ is epi, see Chapter 7 in [8].

In general, any robust approach to the Problem 1.3 should reflect structural properties of $p \in \mathcal{P}\left({ }^{m} E ; E\right)$ such as unboundedness and lack of a vector space structure of $\mathcal{N}(\lambda I-p)$, noncommutativity of $p \in \mathcal{P}\left({ }^{m} E ; E\right)$ with $\lambda I-p$ or generally lin $\left\{p^{n}: n \in \mathbb{N}\right\}$, and the homogeneity degree of $p \in \mathcal{P}\left({ }^{m} E ; E\right)$, and one must cater for the classical notion of eigenvalues in the sense of linear operators. The unboundedness of eigenvalues of the pair $(p, I)$ arises from their structural differences, particularly homogeneity degrees, and the nondiscreteness of their spectrum is due to the lack of a Fredholm alternative or broadly an application of Borsuk's theorem for odd linear and nonlinear maps; see [9]. These pitfalls, except for the unboundedness of $\mathcal{N}\left(\lambda J_{m}-p\right)$, in certain cases can be bypassed while preserving the structure of $p \in \mathcal{P}\left({ }^{m} E ; E\right)$ by replacing the pair $(p, I)$ with $\left(p, J_{m}\right)$ where $J_{m}$ is some well behaved function in the sense that it allows the properties of the pair $\left(p, J_{m}\right)$ to be made compatible; $J_{m}$ may be chosen to cater for the disparity in homogeneity degrees and discreteness of the spectrum of the pair $\left(p, J_{m}\right)$. Specifically, we will choose the function $J_{m}$ to be the homeomorphism defined by $J_{m}(z)=\|z\|^{m-1} z$ where $m$ is the homogeneity degree of $p \in \mathcal{P}\left({ }^{m} E ; E\right)$, and we study Problem 1.3 via the modified eigenvalue Problem 3.1.

Problem 3.1 Given a bounded $p \in \mathcal{P}\left({ }^{m} E ; E\right)$, does there exist a nonzero vector $z \in E$ such that $p(z)=\lambda J_{m}(z)$ for some $\lambda \in \mathbb{k}=\mathbb{C}$ or $\mathbb{R}$ ?

Remark 3.2 Problems 3.1 and 1.3 have negative answers if $E$ is real. Consider the operator $p \in \mathcal{P}\left({ }^{3} \mathbb{R}^{2} ; \mathbb{R}^{2}\right)$ defined by $p\left(x_{1}, x_{2}\right)=\left(-x_{2}^{3}, x_{1}^{3}\right)$. We need to determine whether the eigenvalue equation $p(x)=\lambda|x|^{2} x$, i.e., $\lambda\left(x_{1}^{2}+x_{2}^{2}\right)\left(x_{1}, x_{2}\right)=\left(-x_{2}^{3}, x_{1}^{3}\right)$ holds for $x=\left(x_{1}, x_{2}\right)$ in $\mathbb{R}^{2}$ is solvable for some $\lambda \in \mathbb{R}$. But this means

$$
p(x)=\lambda|x|^{2} x \Rightarrow\left\{\begin{array}{l}
\lambda x_{1}^{3}+\lambda x_{2}^{2} x_{1}=-x_{2}^{3}, \\
\lambda x_{1}^{2} x_{2}+\lambda x_{2}^{3}=x_{1}^{3} .
\end{array}\right.
$$

Without loss of generality, let $x_{2} \neq 0$ so that

$$
\left\{\begin{array} { l } 
{ \lambda x _ { 1 } ^ { 3 } + \lambda x _ { 2 } ^ { 2 } x _ { 1 } = - x _ { 2 } ^ { 3 } , } \\
{ \lambda x _ { 1 } ^ { 2 } x _ { 2 } + \lambda x _ { 2 } ^ { 3 } = x _ { 1 } ^ { 3 } }
\end{array} \Rightarrow \left\{\begin{array}{l}
\lambda y^{3}+\lambda y+1=0 \\
\lambda y^{2}+\lambda=y^{3}
\end{array}\right.\right.
$$


where $y=\frac{x_{1}}{x_{2}}$. Now write the second equation in equation (3.2) as $\lambda y^{2}=y^{3}-\lambda$ to get

$$
\lambda y^{3}+\lambda y+1=y\left(\lambda y^{2}\right)+\lambda y+1=y\left(y^{3}-\lambda\right)+\lambda y+1=y^{4}+1 .
$$

Therefore, the first equation in equation (3.2) simplifies to $y^{4}+1=0$ whose roots are $\pm \frac{1}{\sqrt{2}}(1+i), \pm \frac{1}{\sqrt{2}}(1-i) \notin \mathbb{R}$. On the other hand, equation (3.2) can be simplified to

$$
\left(1+y^{2}\right) \lambda^{2}+y \lambda+1=0
$$

Now if $x_{1}=0$ then $y=0$ and equation (3.3) reduces to $\lambda^{2}+1=0$, which cannot be solved for $\lambda \in \mathbb{R}$. Further, it is clear that if $x_{1} \neq 0$ then $y \neq 0$ and $\lambda=\frac{-y \pm i \sqrt{4+3 y^{2}}}{2\left(1+y^{2}\right)}$ cannot be real valued so that Problems 1.3, 3.1 have negative answers.

Remark 3.3 The choice $J_{m}(z)=\|z\|^{m-1} z$ is guided by the fundamental idea that any eigenvalue of a linear operator $L \in \mathcal{L}(E)$ associated to an eigenvector $z$ is also an eigenvalue of $L \in \mathcal{L}(E)$ associated to an eigenvector $y=\|z\|^{-1} z$.

Lemma 3.4 below is the modified form of Lemma 2.6 in [3].

Lemma 3.4 Let $p \in \mathcal{P}\left({ }^{m} E ; E\right)$ be associated to the given bounded operator $T \in \mathcal{L}\left({ }^{m} E ; E\right)$ where $\operatorname{dim}(E) \geq 2$. If for some $\lambda \in \mathbb{k}=\mathbb{C}$ or $\mathbb{R}, 0 \neq v \in E$, and $p(v)=\lambda J_{m}(v)$ then $\operatorname{lin}\{v\}$ is invariant for $p \in \mathcal{P}\left({ }^{m} E ; E\right)$.

Proof It suffices to show for any $0 \neq v \in E$ satisfying the hypothesis of the underlying lemma, then also $p(u) \in \operatorname{lin}\{v\}$ whenever $u \in \operatorname{lin}\{v\}$. First, observe that $\operatorname{lin}\left\{\frac{v}{\|v\|}\right\}=\operatorname{lin}\{v\}$ and $p(v)=\lambda\|v\|^{m-1} v$ if and only if $p\left(\frac{v}{\|v\|}\right)=\lambda \frac{v}{\|v\|}$. So, the proof is straightforward since if $p \in \mathcal{P}\left({ }^{m} E ; E\right)$ and $u \in \operatorname{lin}\left\{\frac{v}{\|v\|}\right\}$, we have $p(u)=p\left(\gamma \frac{v}{\|v\|}\right)=\frac{\gamma^{m}}{\|v\|^{m}} p(v)=\frac{\gamma^{m}}{\|v\|^{m}} \lambda\|v\|^{m-1} v=$ $\gamma^{m} \lambda \frac{v}{\|v\|} \in \operatorname{lin}\left\{\frac{v}{\|v\|}\right\}$.

The modification of the pair $(p, I)$ with the pair $\left(p, J_{m}\right)$ for $m \in\{2 n-1: n \in \mathbb{N}\}$ yields the following particular form of Theorem 4 in [18] (also see Theorem 9.12 in [8]).

Theorem 3.5 Let $p \in \mathcal{P}\left({ }^{m} E ; E\right)$ be compact and $E$ be real. Then for $m \in\{2 n-1: n \in \mathbb{N}\}$, $\sigma_{\mathrm{FMV}}\left(p, J_{m}\right) \backslash\{0\}=\sigma_{\mathrm{AGV}}\left(p, J_{m}\right) \backslash\{0\}=\sigma_{F}\left(p, J_{m}\right) \backslash\{0\}=\Phi\left(p, J_{m}\right) \backslash\{0\}=\phi\left(p, J_{m}\right) \backslash\{0\}=$ $\sigma_{\text {ev }}\left(p, J_{m}\right) \backslash\{0\}$ where $\sigma_{\text {ev }}\left(p, J_{m}\right):=\left\{\lambda: \mathcal{N}\left(\lambda J_{m}-p\right) \neq\{0\}\right\}$.

In the situation of Theorem 3.5, the spectrum and resolvent set of the pair $\left(p, J_{m}\right)$ are denoted by $\sigma\left(p, J_{m}\right)$ and $\rho\left(p, J_{m}\right)=\mathbb{C} \backslash \sigma\left(p, J_{m}\right)$ respectively. In general, the spectral radius, $r\left(p, J_{m}\right):=\sup \left\{|\lambda|: \mathcal{N}\left(\lambda J_{m}-p\right) \neq\{0\}\right\}$ and in our case, $r\left(p, J_{m}\right):=\sup \left\{|\lambda|: \lambda \in \sigma\left(p, J_{m}\right)\right\}$. We will say $\sigma_{\text {ev }}\left(p, J_{m}\right)$, the set of eigenvalues of the pair $\left(p, J_{m}\right)$, is bounded if $r\left(p, J_{m}\right)<\infty$ and $T \in \mathcal{L}\left({ }^{m} E ; E\right)$ is quasinilpotent if $r\left(p, J_{m}\right)=0$.

Theorem 3.6 is a consequence of Theorem 3.5

Theorem 3.6 Let $E$ be real and $p \in \mathcal{P}\left({ }^{m} E ; E\right)$ be compact. Then for $m \in\{2 n-1: n \in \mathbb{N}\}$, every $\lambda \in \sigma\left(p, J_{m}\right) \backslash\{0\}$ is an eigenvalue of $p \in \mathcal{P}\left({ }^{m} E ; E\right)$.

Theorem 3.6 demonstrates that certain choices of the pair $\left(p, J_{m}\right)$ yield spectra with desirable properties analogous to the classical spectra of linear maps. The reason for this 
nice behavior is that the local, asymptotic, and global properties of the pair $\left(p, J_{m}\right)$ with equal homogeneity degrees are the same.

Theorem 3.7 Let $E$ be an infinite dimensional real Banach space and $p \in \mathcal{P}\left({ }^{m} E ; E\right)$ be associated to the compact operator $T \in \mathcal{L}\left({ }^{m} E ; E\right)$ for $m \in\{2 n+1: n \in \mathbb{N}\}$. If $\inf _{x \in \partial \Omega}\|p(x)\|>$ 0 and 0 is contained in an open subset $\Omega$ of $E$, then $T \in \mathcal{L}\left({ }^{m} E ; E\right)$ has nontrivial invariant subspaces in $E$.

Proof By the Birkhoff-Kellogg theorem [8], Theorem 10.2, there is $0 \neq \lambda \in \sigma\left(p, J_{m}\right)$. The assertion follows from Theorem 3.6, Lemma 3.4, and Remark 1.2.

\section{Challenges to tackling ISPs for nonlinear operators}

The difficulties associated with Problem 1.3 are mainly structural and this section gives their in-depth understanding by means of concrete examples. The ideal $\mathcal{P}_{f}\left({ }^{m} E ; E\right)$, generated by polynomials maps of the form $p(z)=\phi(z)^{n} b$ where $\phi \in E^{\prime}$ and $b \in E$, is appropriate for this purpose.

\subsection{Lack of a linear vector space structure of a nullset of a nonlinear operator}

Example 4.1 below shows the nullset $\mathcal{N}(\lambda I-p)$ possesses properties that are entirely different from those of linear operators. For example, $\mathcal{N}(\lambda I-p)$ is not always a closed linear vector subspace of $E$; these present difficulties to the construction of particularly strongly invariant subspaces for nonlinear operators.

Example 4.1 Let $p \in \mathcal{P}\left({ }^{3} \mathbb{R}^{2} ; \mathbb{R}^{2}\right)$ be defined by $p(x, y)=\left(p_{1}(x, y), p_{2}(x, y)\right): p_{i}(x, y)=x^{3}-$ $\frac{3}{2} x^{2} y-\frac{3}{2} x y^{2}+y^{3}$ for $i=1,2$. Then clearly, $(1,2),(2,1) \in \mathcal{N}(p):=\left\{(x, y) \in \mathbb{R}^{2}: p(x, y)=0\right\}$ but $(1,2)+(2,1) \notin \mathcal{N}(p)$. So $\mathcal{N}(p)$ is not a linear vector subspace of $\mathbb{R}^{2}$.

\subsection{Lack of eigenvalues}

Theorem 4.2 illustrates the existence of quasinilpotent nonlinear operators even on complex Banach spaces.

Theorem 4.2 Let $E=C[0,1]$. The integral operator $p \in \mathcal{P}\left({ }^{m} E ; E\right)$ defined by

$$
(p(f))(t):=\int_{0}^{t} f(s)^{m} d s
$$

is quasinilpotent. Moreover, $p \in \mathcal{P}\left({ }^{m} E ; E\right)$ does not have eigenvalues.

Proof We tacitly assume $m \geq 2$ since for $m=1$ is the well-known Volterra map and besides the proof for compactness of $p \in \mathcal{P}\left({ }^{m} E ; E\right)$ follows the same arguments as for the Volterra map; see [19], p.248. Consider the $n$-fold iterates of $p \in \mathcal{P}\left({ }^{m} E ; E\right)$;

$$
\begin{aligned}
\left(p^{n}(f)\right)(t) & =\int_{0}^{t}\left(\int_{0}^{t_{n-1}} \cdots\left(\int_{0}^{t_{2}}\left(\int_{0}^{t_{1}} f(r)^{m} d r\right)^{m} d t_{1}\right)^{m} \cdots d t_{n-2}\right)^{m} d t_{n-1}, \\
\left\|\left(p^{n}(f)\right)\right\| & \leq\|f\|^{m^{n}} \int_{0}^{t}\left(\int_{0}^{t_{n-1}} \cdots\left(\int_{0}^{t_{2}}\left(\int_{0}^{t_{1}} d r\right)^{m} d t_{1}\right)^{m} \cdots d t_{n-2}\right)^{m} d t_{n-1} \\
& =\frac{t^{\left(m^{n}+\cdots+m+1\right)}\|f\|^{m^{n}}}{(m+1)^{m^{n-1}}\left(m^{2}+m+1\right)^{m^{n-2}} \cdots\left(m^{n-1}+\cdots+m+1\right)} \\
& \leq\|f\|^{m^{n}}\left((m+1)^{m^{n-1}}\left(m^{2}+m+1\right)^{m^{n-2}} \cdots\left(m^{n-1}+\cdots+1\right)\right)^{-1} .
\end{aligned}
$$


The eigenvalue equation $p(f)=\lambda J_{m}(f)$ gives $p^{n}(f)=\lambda^{\frac{m^{n}-1}{m-1}}\|f\|^{m^{n}-1} f$ and so

$$
|\lambda|^{\left.\right|^{m^{n}-1}} \leq\left((m+1)^{m^{n-1}}\left(m^{2}+m+1\right)^{m^{n-2}} \cdots\left(m^{n-1}+\cdots+m+1\right)\right)^{-1} .
$$

Hence, $|\lambda| \leq \limsup _{n \rightarrow \infty}\left(m^{m^{n-1}} m^{2 m^{n-2}} \cdots m^{n-1}\right)^{-\frac{1}{m^{n-1}}} \rightarrow 0$ since $m^{m^{n}} \rightarrow \infty$ faster than $m^{n} \rightarrow \infty$ as $n \rightarrow \infty$. Therefore, $r\left(p, J_{m}\right):=\sup \left\{|\lambda|: \mathcal{N}\left(\lambda J_{m}-p\right) \neq\{0\}\right\}=0$.

Up to this end, we have shown $p \in \mathcal{P}\left({ }^{m} E ; E\right)$ is quasinilpotent. It now remains to show $0 \in \sigma_{\mathrm{ev}}\left(p, J_{m}\right)$, i.e., $p(f)=0$ if and only if $f \equiv 0$. Now, if $p(f)=0$ then also its derivative $p(f)^{\prime}=0$. But, by equation (4.1), $p(f)^{\prime}=f^{m}=0$, i.e., $f \equiv 0$.

Theorem 4.3 Let $E=C[0,1]$. The integral operator $T \in \mathcal{L}\left({ }^{m} E ; E\right)$,

$$
T\left(f_{1}, \ldots, f_{m}\right)(t)=\int_{0}^{t} f_{1}(s) \cdots f_{m}(s) d s
$$

is quasinilpotent and has nontrivial strongly invariant subspaces in $E$.

Proof Observe the quasinilpotency of the given operator in equation (4.2) is an immediate consequence of Theorem 4.2. Further, consider the operator $p \in \mathcal{P}\left({ }^{m} E ; E\right)$ defined in (4.1) associated to the given integral operator in equation (4.2) and mimicking the proof of Theorem 3 in [14] then clearly $p \in \mathcal{P}\left({ }^{m} E\right.$; $\left.E\right)$ has nontrivial invariant subspaces of the form $\mathcal{M}_{a}=\{f \in C[0,1]: f(s)=0, s \in[0, a] \subset[0,1]\}$ so that by equation (4.2), we easily conclude $T\left(\mathcal{M}_{a}, \ldots, \mathcal{M}_{a}\right) \subseteq \mathcal{M}_{a}$

\subsection{Unbounded or infinite spectral set}

Example 4.4 Let $p \in \mathcal{P}\left({ }^{2} \ell_{2} ; \ell_{2}\right)$ be a positively 2-homogeneous polynomial where $p\left(s_{k}\right) \mapsto$ $\left(s_{k}^{2}\right)$ on $\ell_{2}$ is defined by $\left(s_{1}, s_{2}, s_{3}, \ldots\right) \mapsto\left(s_{1}^{2}, s_{2}^{2}, s_{3}^{2}, \ldots\right)$. Write $s=\left(s_{1}, s_{2}, \ldots\right)$. The generalized eigenvalue equation $p(s)=\lambda J_{2}(s)$ gives

$$
|\lambda|=\left(\sum_{i=1}^{\infty} s_{i}^{4}\right)^{\frac{1}{2}}\left(\sum_{i=1}^{\infty} s_{i}^{2}\right)^{-1}
$$

so that $0<|\lambda| \leq 1$; the case $\lambda=1$ gives $n e_{n} \in \mathcal{N}\left(J_{2}-p\right)$ where $e_{n}=(0, \ldots, 0,1,0, \ldots)$; clearly, $\mathcal{N}\left(J_{2}-p\right)$ is an unbounded infinite set since $\left\|n e_{n}\right\| \rightarrow \infty$ as $n \rightarrow \infty$.

Remark 4.5 Example 4.4 is a representative of the general case; $\mathcal{N}\left(\lambda J_{m}-p\right)$ for all $p \in$ $\mathcal{P}\left({ }^{m} E ; E\right)$ is trivially a cone, so it is unbounded/infinite unless it is $\{0\}$.

Remark 4.6 Example 4.1, Theorem 4.2 and Remark 4.4 exhibit certain extreme properties of $\sigma_{\mathrm{ev}}\left(p, J_{m}\right)$ and $\mathcal{N}\left(\lambda J_{m}-p\right)$. Namely, $\sigma_{\mathrm{ev}}\left(p, J_{m}\right)$ is bounded and $\mathcal{N}\left(\lambda J_{m}-p\right)$ is not a vector space; $\sigma_{\mathrm{ev}}\left(p, J_{m}\right)$ is empty and $\mathcal{N}\left(\lambda J_{m}-p\right)$ is trivial; $\sigma_{\mathrm{ev}}\left(p, J_{m}\right)$ is bounded and $\mathcal{N}\left(\lambda J_{m}-p\right)$ is unbounded/infinite, respectively. Notice $\mathcal{N}\left(\lambda J_{m}-p\right)$ may be unbounded/infinite even for $J_{1}=I$. This shows the bad behavior is not due to the difference in the homogeneity degrees of the pair $\left(p, J_{m}\right)$ but ingrained in the structure of $p \in \mathcal{P}\left({ }^{m} E ; E\right)$.

The underlying extreme properties present different levels of difficulties as well as reflect unprecedented challenges that drastically limit the existing techniques to investigate Problem 1.3 via Problem 3.1 or generally elementary invariant subspace. More precisely, 
Lemma 3.4 cannot be applied to certain nonlinear operators such as the one in Theorem 4.2. Moreover, the lack of a nonlinear analog of Gelfand's formula for computing spectral radius, see Examples 6.5, 7.8 in [8], coupled with the lack of the representation of resolvent of the pair $\left(p, J_{m}\right)$ further aggravates the problem. This arises from the general fact that the construction of elementary invariant subspaces for $p \in \mathcal{P}\left({ }^{m} E ; E\right)$ faces nontrivial difficulties since if $p_{1}, p_{2} \in \operatorname{lin}\left\{p^{n}: n \in \mathbb{N}\right\}$ we might have $p_{1} \circ p_{2} \notin \operatorname{lin}\left\{p^{n}: n \in \mathbb{N}\right\}$.

Conjecture 4.7 Let $p \in \mathcal{P}\left({ }^{m} E ; E\right)$ be associated to $T \in \mathcal{L}\left({ }^{m} E ; E\right)$. The subspace $\overline{\ln }\left\{p^{n}(x)\right.$ : $n \in \mathbb{N}\}$ for a fixed $x \in E$ is invariant for $p \in \mathcal{P}\left({ }^{m} E ; E\right)$ if and only if it is strongly invariant for $T \in \mathcal{L}\left({ }^{m} E ; E\right)$.

In general, Example 4.1, Remark 3.2 and Theorem 4.3 demonstrate that $T \in \mathcal{L}\left({ }^{m} E ; E\right)$ may not have nontrivial invariant subspaces in $E$ if its associated operator $p \in \mathcal{P}\left({ }^{m} E ; E\right)$ is either homotopic to a scalar operator or if $\sigma_{\mathrm{ev}}\left(p, J_{m}\right)$ is empty.

\section{Results and discussion}

In this section, we limit the study of Problem 1.3 via Problem 3.1 to the ideals of finite type generated by $\left\{T_{1} T_{2} \ldots T_{m}: T_{j} \in \mathcal{L}(\mathcal{A})\right\}$ where $m \in \mathbb{N}$ is fixed and $\mathcal{A}$ is a Banach algebra. This ideal class is larger than $\mathcal{P}_{f}\left({ }^{m} \mathcal{A} ; \mathcal{A}\right)$ and the ideals coincide only if $\mathcal{A}$ has an $r_{n}$-property, see [20]; moreover, if $T_{j} \in \mathcal{L}(\mathcal{A})$ are linear endomorphisms then the nonlinear operators they generate are known to preserve the structures of Banach algebras, a property most suitable for our research framework.

The literature in [21], Chapter VI and [22], Chapters 10, 11, on Banach algebras is sufficient for our purpose. A normed linear complex space $(\mathcal{A},\|\cdot\|)$ equipped with a multiplication $(a, b) \rightarrow a b$ from $\mathcal{A} \times \mathcal{A}$ into $\mathcal{A}$ is a normed algebra if it is an algebra and $\|a b\| \leq\|a\|\|b\|$ for all $a, b \in \mathcal{A}$. A normed algebra $\mathcal{A}$ is a Banach algebra if the normed space $(\mathcal{A},\|\cdot\|)$ is a Banach space. A Banach algebra $\mathcal{A}$ is commutative if $a b=b a$ for all $a, b \in \mathcal{A}$; it is unital if there is $1 \in \mathcal{A}$ such that $1 a=a=a 1$ for all $a \in \mathcal{A}$ and such an element is called a unit. Here, we take the unit $1 \in \mathcal{A}$ to have norm one. An element $a \in \mathcal{A}$ is called an idempotent if $a^{2}=a$.

A vector subspace $\mathcal{B}$ of $\mathcal{A}$ is a subalgebra if itself is an algebra with respect to the operations of $\mathcal{A}$. A subalgebra $\mathcal{U}$ of an algebra $\mathcal{A}$ is a right ideal if $\mathcal{U} \mathcal{A}=\{u a: u \in \mathcal{U}, a \in \mathcal{A}\} \subset \mathcal{A}$ and it is a left ideal if $\mathcal{A U}=\{a u: u \in \mathcal{U}, a \in \mathcal{A}\} \subset \mathcal{A}$; it is a two-sided ideal if it is both left and right ideal. A proper ideal $\mathcal{M} \subset \mathcal{A}$ is called a maximal ideal if it is not properly contained in any other proper ideal. In a commutative unital algebra every proper ideal is contained in some maximal ideal and the same holds for noncommutative algebras with the observation that left and right ideals should be treated separately (the corresponding maximal ideals are also left or right); the proofs of these facts are premised on Zorn's lemma.

Definition 5.1 An operator $T: \mathcal{A} \rightarrow \mathcal{A}$ where $\mathcal{A}$ is a unital algebra is unital if $T(1)=1$, otherwise, it is nonunital.

Remark 5.2 The Gelfand-Mazur theorem, see Chapter VI in [21], Theorem 1.3, is key in understanding structural aspects of algebras and thus the study of Problem 1.3. Indeed, it is known that $\mathbb{C}$ is isomorphic to $\mathbb{R}^{2}$ and ISP for $T \in \mathcal{L}\left({ }^{m} \mathbb{R}^{2} ; \mathbb{R}^{2}\right)$ has been solved affirmatively, see Theorems 4.5, 4.9 in [3]. On the one hand, the existence of isomorphisms between $\mathbb{C}$ and $\mathbb{R}^{2}, \mathbb{C}$, and the algebra $\mathcal{A}$ whose every nonzero element is invertible allows 
one to easily extend the known ISP results for $T \in \mathcal{L}\left({ }^{m} \mathbb{R}^{2} ; \mathbb{R}^{2}\right)$ to $T \in \mathcal{L}\left({ }^{m} \mathcal{A} ; \mathcal{A}\right)$, since an isomorphism preserves invariant subspaces and is also key in developing techniques for tackling Problem 1.3; see Remark 4.11 in [3]. Therefore, what remains to be considered are the algebras that are not isomorphic to $\mathbb{C}$.

Notation 5.3 Hereafter, all operators will be assumed to be continuous. Let $T \in \mathcal{L}(\mathcal{A})$ be a bounded linear operator on a complex Banach algebra $\mathcal{A}$ and $p \in \mathcal{P}\left({ }^{2} \mathcal{A} ; \mathcal{A}\right)$ be associated to $Q \in \mathcal{L}\left({ }^{2} \mathcal{A} ; \mathcal{A}\right)$. Let $E_{p}:=\left\{0 \neq z \in E: p(z)=\lambda J_{2}(z)\right.$ for some $\left.\lambda \in \mathbb{C}\right\}$ be the set of eigenvectors of the pair $\left(p, J_{2}\right)$ where $J(z)=\|z\| z$ and let $E_{T}:=\{0 \neq z \in E: T(z)=\lambda z$ for some $\lambda \in \mathbb{C}\}$ be the set of eigenvectors of $T \in \mathcal{L}(\mathcal{A})$.

Theorem 5.4 Let $\mathcal{A}$ be a complex unital Banach algebra and $T \in \mathcal{L}(\mathcal{A})$ be a linear operator. The bilinear operator $Q \in \mathcal{L}\left({ }^{2} \mathcal{A} ; \mathcal{A}\right)$ defined by $T(z, w)=z T(w)$ for all $z, w \in \mathcal{A}$ has nontrivial invariant subspaces in $\mathcal{A}$.

Proof We will tacitly assume that the algebra $\mathcal{A}$ is infinite dimensional since the result is already known in finite dimensions; see [3]. Let $p \in \mathcal{P}\left({ }^{2} \mathcal{A} ; \mathcal{A}\right)$ be the polynomial operator associated to the given bilinear operator $Q \in \mathcal{L}\left({ }^{2} \mathcal{A} ; \mathcal{A}\right)$; this polynomial operator is defined by $p(z)=z T(z)$ for all $z \in \mathcal{A}$. Now, if $p \in \mathcal{P}\left({ }^{2} \mathcal{A} ; \mathcal{A}\right)$ is unital then the linear operator $T \in \mathcal{L}(\mathcal{A})$ is also unital and so $1 \in E_{p} \cap E_{T}$. Thus, by Lemma 2.6 in $[3], p \in \mathcal{P}\left({ }^{2} \mathcal{A} ; \mathcal{A}\right)$ and $T \in \mathcal{L}(\mathcal{A})$ have at least a common nontrivial invariant subspace in $\mathcal{A}$ and subsequently by Remark 1.2 , the given bilinear operator $Q \in \mathcal{L}\left({ }^{2} \mathcal{A} ; \mathcal{A}\right)$ has at least a nontrivial strongly invariant subspace in $\mathcal{A}$.

On the other hand, suppose $p \in \mathcal{P}\left({ }^{2} \mathcal{A} ; \mathcal{A}\right)$, hence $T \in \mathcal{L}(\mathcal{A})$, is nonunital. Now, pick a noninvertible element $0 \neq a \in \mathcal{A}$ and fix it in $\mathcal{A}$; this element, as a consequence of the Gelfand-Mazur theorem, see Chapter VI in [21], Theorem 1.3, exists in $\mathcal{A}$. Then to prove the underlying theorem, it first suffices to show $\bar{I}_{a}$, the closure of the ideal $I_{a}:=\{a b: b \in \mathcal{A}\}$, is nontrivial and invariant for $p \in \mathcal{P}\left({ }^{2} \mathcal{A} ; \mathcal{A}\right)$. First, by the noninvertibility of $a \in \mathcal{A}, 1 \notin I_{a}$ and, moreover, it is a known fact that the closure of a proper ideal of a unital algebra is proper, see Corollary 5.2 in [23]; so, to ascertain that $I_{a}$ and its closure $\bar{I}_{a}$ are proper in $\mathcal{A}$ it is enough to show $1 \notin \bar{I}_{a}$. Now, by way of contradiction, suppose $1 \in \bar{I}_{a}$. Then one can find an element $x \in I_{a}$ such that $\|1-x\| \leq \frac{1}{2}$. But, by a Neumann series expansion, see Theorem 10.7 in [22], $x=1-(1-x)$ is invertible so that $1=x^{-1} x=x x^{-1} \in I_{a}$, a contradiction. It now remains to show that $\bar{I}_{a}$, the closure of $I_{a}$, is invariant for $p \in \mathcal{P}\left({ }^{2} \mathcal{A} ; \mathcal{A}\right)$. Consider an element $y \in I_{a}$ (i.e., $y:=a b, b \in \mathcal{A}$ ). Then it is straightforward $p(y)=p(a b)=a b T(a b) \in I_{a}$ and the fact that $p: \bar{I}_{a} \rightarrow \bar{I}_{a}$ follows from the known fact that a continuous map that maps a subset of a metric (or a topological) space into another metric (topological) space maps the closures of the underlying subsets from one into the other, see p.103, Theorem 7.1 in [24]. By Remark 1.2, $Q \in \mathcal{L}\left({ }^{2} \mathcal{A} ; \mathcal{A}\right)$ has a nontrivial closed invariant subspace in $\mathcal{A}$.

Remark 5.5 Notice the polynomial operator defined in Theorem 5.4 can be viewed as a pointwise product of an identity operator $I \in \mathcal{L}(\mathcal{A})$ and a linear operator $T \in \mathcal{L}(\mathcal{A})$; moreover, $I \in \mathcal{L}(\mathcal{A})$ is an isomorphism. This observation leads to the following more general situations embodied in Theorems 5.6, 5.7 below.

Theorem 5.6 Let $\mathcal{A}$ be a complex unital Banach algebra and $S, T \in \mathcal{L}(\mathcal{A})$ be linear endomorphisms. The bilinear operator $Q \in \mathcal{L}\left({ }^{2} \mathcal{A} ; \mathcal{A}\right)$ defined by $Q(z, w)=S(z) T(w)$ for all $z, w \in \mathcal{A}$ has nontrivial invariant subspaces in $\mathcal{A}$. 
Proof Let $p \in \mathcal{P}\left({ }^{2} \mathcal{A} ; \mathcal{A}\right)$ be the polynomial operator associated to the given bilinear operator $Q \in \mathcal{L}\left({ }^{2} \mathcal{A} ; \mathcal{A}\right)$; this polynomial operator is defined by $p(z)=S(z) T(z)$ for all $z \in \mathcal{A}$. Now if the operators $S, T \in \mathcal{L}(\mathcal{A})$ are unital then so is $p \in \mathcal{P}\left({ }^{2} \mathcal{A} ; \mathcal{A}\right)$ so that $1 \in E_{p} \cap E_{T} \cap E_{S}$. Therefore, by Lemma 3.4, $S, T \in \mathcal{L}(\mathcal{A})$, and $p \in \mathcal{P}\left({ }^{2} \mathcal{A} ; \mathcal{A}\right)$ have at least a common nontrivial invariant subspace in $\mathcal{A}$ and by Remark 1.2, the given bilinear operator $Q \in \mathcal{L}\left({ }^{2} \mathcal{A} ; \mathcal{A}\right)$ has a nontrivial invariant subspace in $\mathcal{A}$.

Assume the polynomial operator $p \in \mathcal{P}\left({ }^{2} \mathcal{A} ; \mathcal{A}\right)$ associated to the given bilinear operator $Q \in \mathcal{L}\left({ }^{2} \mathcal{A} ; \mathcal{A}\right)$ is nonunital. So, at least one of the linear operators say $S \in \mathcal{L}(\mathcal{A})$ is also nonunital. Let $X=S(1)$ and consider $\bar{I}_{X}$, the closure of the ideal $I_{X}:=\{X b: b \in \mathcal{A}\}$ in $\mathcal{A}$. Notice the fact that $S \in \mathcal{L}(\mathcal{A})$ is an endomorphism shows that $X=S(1)$ is an idempotent so that it is noninvertible. Subsequently, all elements of $I_{X}$ are noninvertible so that $1 \notin \mathcal{A}$ and, as in Theorem 5.4, both the ideal $I_{X}$ and its closure $\bar{I}_{X}$ are proper in $\mathcal{A}$. We will now show $\bar{I}_{X}$ is invariant for $p \in \mathcal{P}\left({ }^{2} \mathcal{A} ; \mathcal{A}\right)$. Now, since $X=S(1)$ is an idempotent, then, for all $X b \in I_{X}$,

$$
\begin{aligned}
p(X b) & =S(X b) T(X b)=S(1 X b) T(X b) \\
& =S(1) S(X b) T(X b)=X S(X b) T(X b) \in I_{X} .
\end{aligned}
$$

Moreover, for the same reason as in Theorem 5.4, it is clear $p: \bar{I}_{X} \rightarrow \bar{I}_{X}$, i.e., the ideal $\bar{I}_{X}$ is nontrivial and invariant for $p \in \mathcal{P}\left({ }^{2} \mathcal{A} ; \mathcal{A}\right)$. By Remark 1.2 , the given bilinear operator $Q \in \mathcal{L}\left({ }^{2} \mathcal{A} ; \mathcal{A}\right)$ has a nontrivial invariant subspace in $\mathcal{A}$.

Theorem 5.7 Let $\mathcal{A}$ be a complex unital Banach algebra, $S \in \mathcal{L}(\mathcal{A})$ be a linear endomorphism and $T \in \mathcal{L}(\mathcal{A})$ be a linear operator. If

(i) $S \in \mathcal{L}(\mathcal{A})$ commutes with $T \in \mathcal{L}(\mathcal{A})$ and

(ii) $T(1)$ is an idempotent,

then the bilinear operator $Q \in \mathcal{L}\left({ }^{2} \mathcal{A} ; \mathcal{A}\right)$ defined by $Q(z, w)=S(z) T(w)$ for all $z, w \in \mathcal{A}$ has nontrivial invariant subspaces in $\mathcal{A}$.

Proof Let $p \in \mathcal{P}\left({ }^{2} \mathcal{A} ; \mathcal{A}\right)$ be associated to the given bilinear homomorphism $Q \in \mathcal{L}\left({ }^{2} \mathcal{A} ; \mathcal{A}\right)$. Then by Theorem 5.6, if $T \in \mathcal{L}(\mathcal{A})$ is a linear endomorphism, $p \in \mathcal{P}\left({ }^{2} \mathcal{A} ; \mathcal{A}\right)$, and $Q \in$ $\mathcal{L}\left({ }^{2} \mathcal{A} ; \mathcal{A}\right)$ has nontrivial invariant subspaces in $\mathcal{A}$. Therefore, assume $T \in \mathcal{L}(\mathcal{A})$ is not an endomorphism. Now, if both $S, T \in \mathcal{L}(\mathcal{A})$ are unital then so is $p \in \mathcal{P}\left({ }^{2} \mathcal{A} ; \mathcal{A}\right)$ so that all these underlying operators have a common nontrivial invariant subspace in $\mathcal{A}$. On the other hand, if $S \in \mathcal{L}(\mathcal{A})$ is nonunital, set $V=S(1)$ and consider the ideal $I_{V}:=\{V b: b \in \mathcal{A}\}$; the proof can then be fashioned in a similar manner as its analogous case in Theorem 5.6. Thus, it now suffices to prove only the case when $T \in \mathcal{L}(\mathcal{A})$ is nonunital and $S \in \mathcal{L}(\mathcal{A})$ is unital. For this case, let $W=T(1)$ and consider $\bar{I}_{W}$, the closure of $I_{W}:=\{W b: b \in \mathcal{A}\}$ in $\mathcal{A}$. Notice $W$ is idempotent so that it is noninvertible and as in Theorem 5.4, the ideal $I_{W}$ and its closure $\bar{I}_{W}$ are proper ideals in $\mathcal{A}$. To accomplish the proof, it first suffices to show $p: \bar{I}_{W} \rightarrow \bar{I}_{W}$. But, by the problem statement,

$$
\begin{aligned}
p(W b) & =S(W b) T(W b)=S(W) S(b) T(W b) \\
& =S(T(1)) S(b) T(W b)=T(S(1)) S(b) T(W b) \\
& =W S(b) T(W b) \in I_{W}
\end{aligned}
$$


for all $W b \in I_{W}$. Therefore, as in Theorem 5.4, it is clear that $p: \bar{I}_{W} \rightarrow \bar{I}_{W}$ and by $\operatorname{Re}-$ mark $1.2, Q \in \mathcal{L}\left({ }^{2} \mathcal{A} ; \mathcal{A}\right)$ has a nontrivial invariant subspace in $\mathcal{A}$.

\subsection{General comments}

The central point of our results, as envisaged in Theorems 5.4,5.7 and 5.6 is that structural properties of operators and spaces are key in the development of techniques for tackling nonlinear ISP. Indeed, an attempt to generalize the techniques [5] to nonlinear invariant subspace Problem 1.3 fails due to the lack of approximation step [5], Statement II. Besides, the application of the methods $[6,25]$ to nonlinear invariant subspace Problem 1.3 is hampered by the challenges outlined in Remark 4.6. More precisely, the unboundedness and non-vector space structure of the set $\mathcal{N}\left(\lambda J_{m}-p\right)$ devoid of the analogs [26], Lemma 10.2, Theorem 10.18, that are key to the techniques in [6]. Similarly, the application of the method [25] to nonlinear invariant subspace Problem 1.3 shows that quasinilpotent compact multilinear operators have nontrivial invariant subset. Further, to determine invariant subspaces for $T \in \mathcal{L}\left({ }^{m} E ; E\right)$ via the modified nonlinear eigenvalue Problem 3.1 generally requires determining common coincidence points of a family of commuting maps $\left\{q_{i} \in \mathcal{P}\left({ }^{m} E ; E\right): q_{i} q_{j}=q_{j} q_{i}\right.$ for all $\left.i, j \in \mathbb{N}\right\}$ with a homeomorphism $J_{m}$; this results into problems associated with $\sigma_{\mathrm{FMV}}\left(p, J_{m}\right)$ spectrum.

Competing interests

The author declares that he has no competing interests.

\section{Author's contributions}

The work as a whole is a contribution of the author.

\section{Acknowledgements}

I would like to thank Professor Dr. Dirk Werner for his stimulating discussions and personal interest during the preparation of this work.

Received: 6 May 2016 Accepted: 29 June 2016 Published online: 11 July 2016

\section{References}

1. Dineen, S: Complex Analysis on Infinite-Dimensional Spaces. Monographs in Mathematics. Springer, London (1990)

2. Mujica, J: Complex Analysis in Banach Spaces. Math. Stud., vol. 120. North-Holland, Amsterdam (1986)

3. Emenyu, J: An invariant subspace problem for multilinear operators on finite dimensional spaces. Topol. Methods Nonlinear Anal. 43, 1-10 (2014)

4. Cahskan, E, Rueda, P: On distinguished polynomials and their projections. Ann. Acad. Sci. Fenn., Math. 37, 595-603 (2012)

5. Aronszajn, N, Smith, KT: Invariant subspaces of completely continuous operators. Ann. Math. 60(2), 345-350 (1954)

6. Lomonosov, Vl: Invariant subspaces for operators commuting with compact operators. Funct. Anal. Appl. 7, 213-214 (1973)

7. Bényi, A, Torres, RH: Compact bilinear operators and commutators. Proc. Am. Math. Soc. 141(10), 3609-3621 (2013)

8. Appell, J, De Pascale, E, Vignoli, A: Nonlinear Spectral Theory. de Gruyter Series in Nonlinear Analysis and Applications, vol. 10. de Gruyter, Berlin (2004)

9. Furi, M, Martelli, M, Vignoli, A: Contributions to the spectral theory for nonlinear operators in Banach spaces. Ann. Mat. Pura Appl. 118, 229-294 (1978)

10. Feng, W: A new spectral theory for nonlinear operators and its applications. Abstr. Appl. Anal. 2, 163-183 (1997)

11. Väth, M: The Furi-Martelli-Vignoli spectrum vs. the phantom. Nonlinear Anal. TMA 47(9), 2237-2248 (2001)

12. Santucci, P, Väth, M: On the definition of eigenvalues for nonlinear operators. Nonlinear Anal. 40, 565-576 (2000)

13. Buryśek, S: On the spectra of nonlinear operators. Comment. Math. Univ. Carol. 11, 727-743 (1970)

14. Donoghue, WF Jr.: The lattice of invariant subspaces of a completely continuous quasinilpotent transformation. Pac. J. Math. 7, 1030-1035 (1957)

15. Macaev, VI: On a class of completely continuous operators. Dokl. Akad. Nauk SSSR 139, 453-551 (1961) (in Russian)

16. Schwartz, JT: Subdiagonalization of operators in Hilbert spaces with compact imaginary part. Commun. Pure Appl. Math. 15, 159-172 (1962)

17. Zsidó, L: Invariant subspaces of compact perturbations of linear operators in Banach spaces. J. Oper. Theory 1 , 225-260 (1979)

18. Giorgieri, E, Appell, J, Väth, M: Nonlinear spectral theory for homogeneous operators. Nonlinear Funct. Anal. Appl. 7(4), 589-618 (2002)

19. Lax, PD: Functional Analysis. Wiley, New York (2002) 
20. Lourenco, ML, Moras, LA: A class of polynomials from Banach spaces into Banach algebra. Publ. Res. Inst. Math. Sci. 37, 521-529 (2001)

21. Beauzamy, B: Introduction to Operator Theory and Invariant Subspaces. North-Holland Mathematical Library, vol. 42 (1998)

22. Rudin, W: Functional Analysis. McGraw-Hill, New York (1973)

23. Zelazko, W: Banach Algebras. Elsevier, Amsterdam (1973)

24. Munkres, JR: Topology: A First Course. Prentice Hall, New Delhi (2000)

25. Michaels, AJ: Hilden's simple proof of Lomonosov's invariant subspace theorem. Adv. Math. 25, 56-58 (1977)

26. Abramovich, YA, Aliprantis, CD: An Invitation to Operator Theory. Graduate Studies in Mathematics, vol. 50. Am. Math. Soc., Providence (2002)

Submit your manuscript to a SpringerOpen ${ }^{\odot}$ journal and benefit from:

- Convenient online submission

- Rigorous peer review

- Immediate publication on acceptance

- Open access: articles freely available online

- High visibility within the field

- Retaining the copyright to your article

Submit your next manuscript at $\boldsymbol{~ s p r i n g e r o p e n . c o m ~}$ 